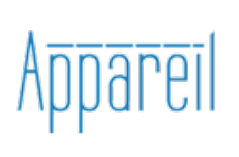

Appareil

$22 \mid 2020$

Une philosophie de l'appareil

\title{
Le Benjamin de Jean-Louis Déotte
}

\section{Alain Brossat}

\section{(2) OpenEdition}

Journals

Édition électronique

URL : http://journals.openedition.org/appareil/3597

DOI : 10.4000/appareil.3597

ISSN : 2101-0714

\section{Éditeur}

MSH Paris Nord

\section{Référence électronique}

Alain Brossat, «Le Benjamin de Jean-Louis Déotte », Appareil [En ligne], 22 | 2020, mis en ligne le 21 décembre 2020, consulté le 26 mars 2021. URL : http://journals.openedition.org/appareil/3597 ; DOI : https://doi.org/10.4000/appareil.3597

Ce document a été généré automatiquement le 26 mars 2021.

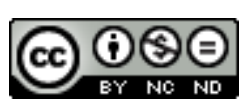

Appareil est mis à disposition selon les termes de la Licence Creative Commons Attribution - Pas d'Utilisation Commerciale - Pas de Modification 4.0 International. 


\title{
Le Benjamin de Jean-Louis Déotte
}

\author{
Alain Brossat
}

1 On voit poindre constamment une tentation dans le livre de Jean-Louis Déotte - celle de la présentation d'un autre Walter Benjamin, qui ne serait pas celui de la réception la plus courante, celle du Benjamin penseur de la politique, de la révolution, de la temporalité messianique, mais un Benjamin caché ou masqué et qui serait avant tout un penseur de la technique. Jean-Louis Déotte ne va pas jusqu'à dire que son Benjamin minoritaire est le vrai Benjamin, mais à certains moments, il n'est pas très éloigné d'une telle (et redoutable) affirmation. C'est le cas, par exemple, à la page 116 de son Walter Benjamin et la forme plastique - architecture, technique, lieux, quand il corrige et réprimande Benjamin en disant que ce dernier fait "un usage trop extensif du terme de dialectique »; un usage qui, en effet, ne trouve pas véritablement sa place dans le tableau benjaminien dessiné par Jean-Louis Déotte mais qui, bel et bien, renvoie à ses affinités avec Bertolt Brecht, donc avec le marxisme révolutionnaire à la Karl Korsch, et qu'il ne saurait être question d'effacer d'un trait de plume. Il en va de même lorsque Déotte insiste non seulement sur le constat que Benjamin est un penseur de la technique «et ce d'une manière non anecdotique, mais essentielle » mais aussi, de surcroît, sur le fait que cet intérêt premier pour la technique, et donc les appareils, constituerait le fil rouge de sa pensée, "depuis les premiers textes (Fragments d'esthétique) jusqu'aux écrits sur Brecht ».

2 Il me semble qu'avec ces formules, Déotte est bien près de succomber à cette tentation tant partagée parmi les commentateurs contemporains d'un philosophe du $\mathrm{xx}^{\mathrm{e}}$ siècle dont l'étoile était en train de monter au firmament de la reconnaissance universitaire (un geste que je rencontre constamment dans la glose qui se développe de nos jours autour de l'œuvre de Michel Foucault), celle qui consiste à écarter d'un geste les lectures antérieures ou en cours, pour dire : me voici, qui arrive à point nommé pour vous dévoiler enfin la vérité de cette œuvre si malmenée jusqu'à présent, une vérité qui se révèle dans la " cohérence et la continuité » jusqu'alors inaperçues de cette pensée.

3 Toutefois, il m'a toujours semblé que s'il y a un auteur qui demeure résolument réfractaire à ce type de réduction, c'est bien Benjamin. Je ne fais pas référence ici uniquement à son geste d'écriture, qui fait de son travail un champ de dispersion et 
d'inachèvement infini, mais tout simplement à ce qui constitue le premier niveau des stratégies d'appropriation de l'œuvre dans sa vérité supposée, c'est-à-dire son niveau philologique - là où les gens de Francfort qui se considèrent comme vicaires de droit de l'œuvre vont rejeter d'un revers de main les lectures françaises de Benjamin, disant «de toute façon, vous n'y comprenez rien, parce que l'allemand de Benjamin est trop compliqué, trop raffiné pour vous ». Or, Maria Muhle (une jeune philosophe allemande) et moi-même nous étions amusés à montrer, il y a quelques années, innombrables traductions à l'appui, que personne, pas même M. Tiedemann, ne peut établir, si ce n'est par décret, ce que la fameuse thèse VIII des Thèses sur la philosophie de l'histoire, veut vraiment dire, pour la bonne raison qu'il y a, au cœur de cette thèse, une phrase qui est grammaticalement indécidable. Ce n'est pas là un problème philologique isolé, c'est un apologue à propos de l'œuvre de Benjamin : il n'y a pas de vérité de l'œuvre, il n'y a que des lectures - ce qui n'exclut évidemment pas que certaines puissent être plus rigoureuses ou, inversement, distraites et opportunistes que d'autres. Cependant et dans le fond, cette position me conduit à refuser de tenter de départager l'ancien du nouveau, les descendants de Karl Marx et Gershom Scholem (Michael Löwy, Daniel Bensaïd, Stéphane Mosès...) des technicistes simondoniens (Jean-Louis Déotte, notamment). À supposer que Benjamin existe comme un auteur et qu'à cet auteur corresponde une cuvre, ce qui se discute, l'un comme l'autre n'existent que dans un champ de dispersion borné, admettons, d'une part par le nom de Gershom Scholem, d'autre part celui de Theodor Adorno, d'autre part celui de Bertolt Brecht, d'autre part celui d'Asja Lācis, etc. Dans ces conditions, chacun est convié à se repérer topographiquement dans son œuvre en y reconnaissant des points ou des lieux d'identification en forme de motifs (la fantasmagorie, le petit bossu, le présent comme catastrophe, le passage, Moscou), de noms propres, de reprises, et à y tracer ses propres lignes de lecture sans jamais pouvoir prétendre revenir à ce que Benjamin a toujours combattu me semble-t-il avec acharnement, dans la figure de l'historien positiviste : le fameux eigentlich - dire ce que le passé a été en vérité, dire ce que l'œuvre de Benjamin est en vérité.

Ceci étant dit, le caractère même du livre de Déotte, construit en mosaïque, même s'il réveille toujours au fond les mêmes pistes de lecture, retient l'auteur de trop succomber à la tentation. Ce n'est pas seulement un livre sur Benjamin et la technique, mais aussi sur Benjamin et la ville, Benjamin et la fantasmagorie, Benjamin et l'enfance, Benjamin et la chaussette, etc. Il s'agit donc d'un ouvrage qui n'est pas lui-même suffisamment systématique pour nous présenter un Benjamin systématisé, ce qui serait aller à l'encontre de toutes les évidences que livre la lecture d'un corpus qui, farouchement, se défend contre le statut d'œuvre même. C'est un opus agréable à lire, inattendu, car c'est un livre de pistes de lecture. Une flânerie parmi les écrits de Benjamin et non pas le plaquage d'une systématique sur le corpus dispersé des écrits de celui-ci.

Je vais donc me contenter de relever quelques-uns de ces motifs, tels qu'ils émergent au cours de cette promenade, en regrettant que Déotte ne puisse plus préciser ou expliciter certaines de ses propositions.

5 Un concept est présenté avec une réelle insistance dans la première partie du livre, celui de distraction. Le rapport de la masse moderne (comme foule) à la ville et à l'architecture (il faudrait aussi mobiliser, pour le présent, la notion d'urbanisme et l'enjeu des stratégies urbanistiques du pouvoir en construisant une passerelle entre 
Walter Benjamin et Henri Lefebvre) est un rapport de distraction. Cette dernière s'oppose ici à la contemplation mais aussi à l'attention, à la conscience de, etc. Dans ce rapport de distraction, la masse ne perçoit pas l'architecture sur un mode objectal, mais est au contraire absorbée par elle. Les fantasmagories prospèrent sur le terreau de cette imprégnation dans laquelle s'abolit la distinction entre un objet de perception et un sujet perceptif. La foule qui vaque à ses occupations, y compris le flâneur qui dérive à travers la ville en laissant flotter son attention, est une éponge qui absorbe toutes sortes de sensations, d'affects, et les met en relation sur un mode plastique avec tout ce qui constitue le domaine d'appareillage du monde - de la ville en l'occurrence. Ce qui reste un peu pendant, dans cette analyse, c'est la condition subjective du vivant urbain, aussi bien celle de la masse que celle du flâneur, du poète, du visiteur, etc. Avant de signifier la distraction, le mot Zerstreuung exprime la dispersion : in alle Winde zerstreut dispersé à tous vents, aux quatre coins de la terre. Ce qui veut dire que l'état subjectif de la masse, mais aussi bien du passant, de l'oisif qui flâne dans le passage, de la prostituée qui arpente le trottoir, du garçon de course qui vaque à ses occupations, n'est pas seulement celui d'un engloutissement par les formes architecturales et la marchandise qui l'entourent, mais également celui d'un éclatement perpétuel, constamment renouvelé sous l'effet de la succession des chocs discontinus produits par sa perception d'une réalité urbaine discontinuée, radicalement placée sous le signe de l'hétérogène.

6 Dans ces conditions, il semblerait que seule une sorte d'inconscient collectif, de transindividualité protoplasmique des profondeurs psychiques collectives puisse faire tenir ensemble ces éclats de subjectivité... Que reste-t-il alors du sujet, dont ici, pour faire référence à un autre passage du livre, il ne subsiste " pas même un nom propre " ? $\mathrm{Si}$, dans la ville moderne, la condition ordinaire du socius est soumise à cet état perpétuel d'éclatement de fragments subjectifs, comment peut-on imaginer la possibilité même d'un passage du social au politique, soit de la masse au public ou bien, dans des contextes plus effervescents, de l'écoulement de la masse sur les trottoirs des boulevards à l'émeute, à la révolution, ce qui suppose bien, dans les deux cas, quelque chose comme la formation d'une subjectivité rassemblée et dotée d'une capacité d'inscrire ses gestes et ses conduites dans un horizon partagé ? Est-ce que la lecture du Livre des passages a dynamité le cartésianisme des origines de Déotte, auquel, il y a quelques années encore, si je me rappelle bien, il tenait assez rigoureusement?

7 Un second enjeu de taille dans cet ouvrage est, me semble-t-il, la mise en perspective de la notion de fantasmagorie. Dans le Livre des passages, rappelle Jean-Louis Déotte, la fantasmagorie ne renvoie pas à un modèle explicatif, mais au contraire à un double parti, descriptif et analytique : en analysant des opérateurs de fantasmagorie, on va tenter de montrer, de l'intérieur plutôt que par une approche objectiviste, «scientifique », comment ça marche, c'est-à-dire comment la marchandise, le capital, exerce ses séductions sur la masse, le passant, le consommateur... En outre, ce choix en faveur du comment plutôt que du pourquoi est un point sur lequel le geste philosophique de Benjamin se rapproche de celui du Foucault de la généalogie et de l'archéologie - une affinité élective que ne peuvent comprendre que ceux qui ne lisent pas Foucault à charge - comme Déotte l'a toujours fait... Comme ce dernier le montre bien, la fantasmagorie, les fantasmagories, c'est le rêve éveillé de celui qui chemine dans les espaces enchantés du capital, de la ville architecturée, des rues, des galeries, des grands magasins à la fin du XIX $x^{e}$ et dans la première moitié $d u x^{e}$ siècle, des centres 
commerciaux actuels (il y aurait une thèse à faire sur la figure du mall dans le cinéma américain contemporain, je pense à George Romero, par exemple) et qui, de la sorte, est absorbé par une forme d'inconscient collectif, lequel est indistinctement celui de la masse et du capital lui-même. L'habitant de la planète marchandise ou capital, dans cette perspective, est moins aliéné (verfremdet, entfremdet), c'est-à-dire littéralement soumis à une puissance étrangère, arraché à son moi originel ou son moi propre par cette puissance) qu'onirisé, dirais-je, si l'on veut bien accepter ce redoutable néologisme. C'est évidemment là que l'on rencontre la question du cinéma, "un appareil configurant si profondément l'expérience et la connaissance qu'il a le statut d'inconscient même pour Benjamin", comme Déotte l'écris si justement - chose dont Edgar Morin a également eu l'intuition dès les années 1950. Ceci a pour effet, si l'on aborde la question sous l'angle ontologique, de montrer que le capital n'est pas une machine à produire de l'illusion, à recouvrir la réalité de couches toujours plus épaisses d'illusion pour nous détourner de la réalité vraie (les plus convaincus des marxistes scolastiques iront jusqu'à dire la réalité concrète). Au contraire, le capital est d'emblée une machine à produire des sujets onirisés (il n'en est pas d'autres sous son règne) et donc une réalité enchantée. Toute réalité du capital est "enchantée »; c'est-à-dire, si l'on suit bien le Benjamin de la fantasmagorie, indistincte du rêve. Le problème n'est donc pas tellement pour nous, qui aspirons à être moins gouvernés par le capital, voire à ne pas l'être du tout, de rejeter cette emprise pour revenir à une supposée réalité vraie qui, à proprement parler, n'existe pas, qui n'est que le revers du fantasme, mais plutôt de tenter de détourner, d'infecter, etc., le grand rêve du capital et d'infléchir constamment notre condition de sujets oniriques (de protagonistes du grand rêve collectif). Il n'est pas question de reprendre pied dans la réalité vraie qui est la rêverie inconsistante par excellence des essentialistes, mais d'apprendre à rêver autrement, à donner une autre destination à notre condition de rêveurs - ce que fait le collectionneur qui « détourne » le rêve du capital, aussi bien qu'Auguste Blanqui, dont le texte sur l'Éternité par les astres montre bien qu'il est le rêveur par excellence, mais dont l'énergie onirique s'est portée sur des objets un peu moins futiles que ceux que proposent les vitrines et les boutiques des passages -, la prise d'armes, la belle insurrection armée.

Cette approche de l'enveloppement ou l'investissement de nos existences par le capital (séduction, donc, autant que domination : sur ce point, Jean Baudrillard a, pour une fois, vu juste) est ce qui permet à Benjamin de donner au mot fantasmagorie un sens extraordinairement ambivalent. Nous sommes sous l'emprise des fantasmagories, mais cette emprise, c'est notre vie. Le flâneur, le visiteur du musée, le spectateur dans la salle de cinéma, le consommateur qui hante le grand magasin et la galerie marchande sont, par définition, des figures ambiguës. Ils subissent les conditions du capital, des stratégies commerciales, des industries culturelles, mais le rêve éveillé dans lequel les plonge cette emprise est leur milieu de vie, le seul terreau sur lequel peuvent surgir des pensées dissidentes, des contre-conduites. C'est l'ambivalence constitutive du cinéma que Benjamin a d'emblée détectée : une machine à abrutir et infantiliser le public, comme l'ont dit depuis le début ses détracteurs, mais aussi bien un formidable terrain d'expérimentation pour la pensée en général et les pensées en quête d'un dehors (par opposition à l'extérieur, qui n'existe pas) de cette « domination » (mais on touche ici les limites du terme domination qui suppose la séparation de ce qui domine et de ce qui est dominé). Or, le problème est précisément, ici, celui de la non-séparation (parlons donc 
plutôt d'emprise, d'investissement, d'infiltration, de capture) : l'envoûteur et l'envoûté deviennent rapidement indissociables et emportés par un même rêve éveillé.

Comme Déotte le dit très bien, p. 60, le mot fantasmagorie, dans son ambivalence même, est ce qui, en s'opposant à la notion d'idéologie, permet à Benjamin de creuser la distance avec le marxisme scolastique. Ce n'est pas un hasard si, dans les usages de ce dernier, la notion d'idéologie a un sens tout entier péjoratif : c'est l'indice probant de la fatale simplification que récuse Benjamin en laissant flotter, lui, le mot fantasmagorie. L'idéologie, en effet, c'est la fausse connaissance, la perception faussée de la réalité, découlant directement des jeux de forces et des intérêts économiques et sociaux. L'idéologie est un produit. Elle est également instrumentale, indissociable des calculs et des stratégies, et trouve sa place dans un édifice où la superstructure résulte de l'infrastructure, en est la conséquence et la représentation aliénée. Benjamin, en rompant avec cette notion dans le Livre des passages, dans la partie "baudelairienne " de son œuvre, du moins (quand il est brechtien, c'est une autre affaire...), met le doigt sur ce qui constitue le pont aux ânes du marxisme scolastique: la théorie de la détermination de la superstructure par l'infrastructure, comme si l'on pouvait distinguer le statut ontologique d'une réalité vraie, matérielle, solide constituant le fondement objectif de la vie vécue des humains d'avec celui d'une fausse réalité faite d'idées, de croyances, de discours qui en constituerait la représentation fallacieuse, agencée aux fins de reproduction de la domination. C'est un rideau de fumée devant la "réalité vraie». Comme si l'architecture métallique de la fin du xIX et les grands travaux de Georges Haussmann n'étaient pas d'emblée un amalgame de fonte et de rêve, c'est-à-dire quelque chose qui ne se décrit bien que dans la langue de la fantasmagorie...

10 En ce sens, on pourrait même radicaliser la proposition de Déotte lorsqu'il écrit que «les rapports entre infrastructure matérielle et superstructure "idéologique" sont d'expression et non de causalité ", jusqu'à récuser la sacro-sainte (ré)partition en infraet superstructure. Qu'est-ce que la tour Eiffel, en effet? Un édifice métallique mobilisant tant de milliers de tonnes de fonte ou le grand rêve d'érection du capital, un glorieux phallus d'acier ? L'un comme l'autre, pas l'un sans l'autre, évidemment.

11 Si la question de l'inconscient collectif était très discutée dans les années 1968, ce n'était pas dans son acception benjaminienne - l'œuvre de Benjamin n'étant pas encore connue -, mais au sens de de l'inconscient collectif fasciste de la masse tel que traité par Wilhelm Reich et Herbert Marcuse. Il s'agit du rapport entre la répression de la sexualité par les dispositifs moraux, familiaux, religieux de la bourgeoisie et l'exutoire constitué par la désublimation répressive proposée par les mouvements fascistes. La montée des différentes orthodoxies analytiques a ensuite radicalement discrédité ce syntagme, ce concept. Une discussion à reprendre, à la lumière de Walter Benjamin et Gilbert Simondon?

Cela ne surprendra personne, la notion d'appareil parcourt l'ensemble de l'essai. Au début, l'accent est porté sur le fait que l'architecture est un élément fondamental, si ce n'est l'élément constitutif de l'appareillage de la ville. On lit p. 35 : «C'est Benjamin qui aura le mieux compris que la ville n'existe pas en soi [...], parce qu'elle est toujours appareillée ». Cependant, l'appareillage ne se manifeste pas seulement du côté des conditions de... (la veine kantienne de la théorie déottienne des appareils), des conditions de la perception, des déplacements dans l'espace urbain, mais aussi du côté de l'actif en entrant en composition dans les formations subjectives. Je relève cette 
formule: "le flâneur apparie de l'hétérogène » et la capacité d'apparier suppose l'appareillage de celui qui apparie l'hétérogène. L'appareillage, donc, est tout autre chose que les déterminations au sens que le marxisme scolastique donne à ce terme. Il suppose la constitution de sujets appareillés/appareillants qui ne vont pas se contenter de rêver ou de fantasmer (fantasmagoriser, plutôt, si l'on veut marquer l'écart avec la théorie freudienne du fantasme) la ville, mais la peupler de leurs conduites et de leurs actions. Comme je le disais plus haut et j'insiste, Blanqui est un rêveur du XIX siècle de Benjamin au même titre que le flâneur, la prostituée et le poète - et Dieu sait pourtant s'il est bien du côté de l'action. Il n'y a pas d'un côté des appareils qui imposeraient des conditions en amont ou dans le dos des sujets et, de l'autre, des sujets qui subiraient ces conditions et devraient s'y adapter; il y a cette capacité spécifique des appareils à faire époque sur un mode tel que l'extériorité supposée des conditions générales ne peut plus être dissociée de l'intériorité des sujets.

13 C'est évidemment là que l'image de la chaussette tend à devenir une métaphore de notre condition générale à l'époque des appareils. Nous sommes tous des paires de chaussettes retournées sur elles-mêmes parce que, comme sujets, nous sommes d'emblée, aussi, la totalité de ces « conditions » qui font de nous les contemporains de notre époque. Après les blessures narcissiques successives que nous ont infligé le darwinisme, la psychanalyse, la théorie de la relativité, le structuralisme, que sais-je encore, nous étions devenus des sujets troués, descellés, destitués, jetés-là, décentrés, etc. Dans l'œuvre de Déotte, lecteur des souvenirs d'enfance de Benjamin, cette déchéance du sujet-roi atteint son paroxysme avec ce devenir-chaussette ou plutôt paire de chaussettes retournées sur elles-mêmes, et voue celui-ci à l'abandon le plus rigoureux - songez à l'emploi du mot chaussette dans les expressions courantes « il s'en soucie comme de sa première paire de chaussettes », « il m'a laissé tomber comme une vieille chaussette! »

14 Je plaisante, mais il me semble que sur ce point, la lecture scrupuleuse de ces textes de Benjamin, souvent considérés comme anecdotiques, ouvre la voie à une critique radicale et très féconde des approches psychologisantes de l'intériorité. Il faudrait aller plus loin dans cette direction en montrant que l'intériorité n'est pas ici ce qui s'oppose à l'extériorité, pas plus que l'intérieur et l'extérieur n'entretiennent des relations dialectiques, que ce n'est pas non plus simplement un enjeu de porosité, comme dans le cas de la membrane chère à Philippe Roy, mais que tout se joue, topographiquement, dans la figure d'un enroulement, d'une torsion insensible dont l'intérieur enchaîne sur l'extérieur et réciproquement. Il faudrait alors être mathématicien pour parler savamment des parentés topologiques du ruban de Möbius et de la paire de chaussettes benjamino-déottienne.

15 Une fois que l'on a relevé cela, tout ne s'est pas éclairci pour autant. Le lecteur attentif de cet ouvrage et des autres écrits de Déotte aura compris que pour ce dernier le passage, le cinéma, sont des appareils constitutifs de l'époque, notre époque. D'où l'importance d'une lecture conjointe du Benjamin du Passagen-Werk et du livre de Siegfried Kracauer, Théorie du film. Ce n'est donc pas un seul appareil qui fait époque, mais une pluralité ou une conjonction d'appareils. Toutefois, sur ce point, l'argumentation devient un peu flottante. On relève, au tout début du livre, la formule suivante : «C'est le passage urbain qui, au XIX siècle est la pièce essentielle [je souligne] de la configuration de la fantasmagorie originaire [je souligne] dont nous héritons », et 
un peu plus loin, celle-ci : "S'il y a aujourd'hui une crise de la ville, une crise de l'urbanisme, c'est que plus aucun appareil n'arrive à s'imposer aux autres ».

Il semble donc qu'il y ait concurrence, lutte pour l'hégémonie, voire guerre, entre les différents appareils qui définissent les conditions d'une époque. À cet égard, nous aimerions en savoir davantage. Lorsque l'on referme le livre, on a bien compris que tout dispositif technique n'a pas vocation à devenir un appareil, de la même façon que tout ce que les journaux appellent « révolution» n'a pas vocation à inscrire la trace d'un événement, mais on ne sait plus très bien dans quel temps des appareils nous sommes. L'âge de la cybernétique dans lequel nous sommes plongés ne se décrit-il pas comme " époque »? L'ordinateur n'est-il qu'un avatar technique dans la topographie d'une modernité dont les conditions demeureraient, fondamentalement, baudelairiennes? Sur ce point, il me semble que l'essai demeure en suspens.

Une question sur laquelle l'ouvrage tranche, en revanche, contre Giorgio Agamben, c'est celle du contemporain, de la contemporanéité, comme l'indique cette formule lapidaire et péremptoire : «Si chaque appareil fait époque et fait monde, c'est lui qui rend les hommes contemporains» (p. 75). La formule est problématique, car on ne voit pas comment chaque appareil, c'est-à-dire un appareil singulier, pourrait faire époque à lui seul s'il est en même temps en lutte ou en concurrence (« n'arrive pas à s'imposer ») avec d'autres... Par ailleurs, cette définition abrupte du contemporain en tant qu'assigné à l'appareil-qui-fait-époque me semble régresser par rapport à d'autres que j'ai mentionnées plus haut et où étaient mise en exergue la question de la subjectivation du présent - le flâneur, en " appariant » de l'hétérogène, active l'appareillage et ouvre un espace dans lequel sa liberté est en question.

Il me semble que l'on ne peut pas statuer si facilement sur le contemporain sans le mettre en relation avec le présent et l'actuel, c'est-à-dire statuer sur ce qui met en relation des conditions générales avec la possibilité pour nous de prendre des positions sur ce qui compte dans le présent - définit les conditions d'une actualité. La formule abrupte de Déotte laisse totalement de côté la question de savoir comment s'agencent l'une sur l'autre notre condition de subjectivité appareillée et notre vocation critique face au présent - l'autre volet du message kantien. On rejoint là un problème récurrent dans la présentation par Déotte de sa théorie des appareils : la tentation perpétuelle de la liquidation de la politique. Autant je serais d'accord avec lui pour dire que la dépréciation de la technique en général n'est pas le meilleur des biais pour rétablir les droits de la politique, autant il me semble qu'une certaine systématicité de la théorie des appareils tend à rendre indistinct l'espace dans lequel la politique, constamment mise à mal par le biopouvoir contemporain, est appelée à se reconstituer. Je ne veux pas du tout dire qu'il s'agirait de défendre bec et ongles une théorie de l'immunité ou de l'intégrité du sujet politique face aux appareils, mais de penser la possibilité envers et contre tout d'une politique, dans le milieu même des appareils. Or, pour aller dans ce sens, il faut opérer un déplacement décisif et volontariste par rapport à la formule de référence - je suis peut-être bien, en première instance, le contemporain de ceux qui voient les mêmes films que moi et sont soumis aux mêmes opérateurs de fantasmagories que moi, mais, au bout du compte, mes vrais contemporains sont ceux avec lesquels je partage une certaine approche de l'actuel, des convictions, des positions, des actions politiques. Si l'on ne dit pas cela, on dit au fond que ce qui fait de nous des contemporains, c'est le seul partage de l'inconscient collectif du capital, notre seule condition commune de rêveurs éveillés. Des zombies, en quelque sorte, avançant 
dans les rues de la ville en somnambules, comme les morts-vivants des films de Romero. En sommes-nous vraiment là ?

\section{RÉSUMÉS}

Dans cette discussion de l'ouvrage Walter Benjamin et la forme plastique - architecture, technique, lieux, publié en 2012 par Jean-Louis Déotte (L'Harmattan, coll. «Esthétiques »), Alain Brossat retrace l'interprétation originale du philosophe allemand par le philosophe français.

\section{INDEX}

Mots-clés : distraction, fantasmagorie, idéologie, politique, contemporain

\section{AUTEURS}

\section{ALAIN BROSSAT}

Research Fellow à l'International Center for Cultural Studies, National Chiao Tung University, Taïwan. 